\title{
APPROXIMATION OF COMMUTING TRANSFORMATIONS
}

\author{
M. A. AKCOGLU AND R. V. CHACON
}

\begin{abstract}
Let $\sigma$ and $\tau$ be two (measure-preserving) transformations. The main purpose of the paper is to show that if $\tau$ admits approximation by partitions and that if $\sigma$ commutes with a power $\tau^{s}$ of $\tau$, then $\sigma$ can be approximated by a finite number of powers of $\tau$. As an application of the result we solve a problem posed earlier, showing that there exist strongly mixing transformations with only a finite number of prescribed roots.
\end{abstract}

1. Introduction. Let $\sigma$ and $\tau$ be two (measure-preserving) transformations of a normalized nonatomic Lebesgue space. The main purpose of this paper is to show that if $\tau$ admits an approximation by partitions (Definition 2.5) and if $\sigma$ commutes with a power $\tau^{s}$ of $\tau$, then $\sigma$ can be approximated by a finite combination of powers of $\tau$ (Theorem 3.1). This result generalizes the main theorems of [1] and [2], which correspond to the special case of $s=1$. The methods are extensions of those introduced in [2] and [3]. As an application, we show that there are strongly mixing transformations with only a finite number of prescribed roots, answering a question raised in $[4$, p. 133].

2. Definitions. Let $(X, \mathscr{F}, \mu)$ be a normalized nonatomic Lebesgue space; i.e. a measure space isomorphic to the unit interval. All subsets of $X$ that are referred to are understood to be in $\mathscr{F}$. In what follows we will recall a few definitions from [1] and [2] to make the present paper selfcontained.

Definition 2.1. A collection $\xi$ of pairwise disjoint sets will be called a partition. The union of the sets in $\xi$ is denoted by $X_{\xi}$, which, in general, is a proper subset of $X$. If $\xi$ is a partition, then $\mathscr{F}(\xi)$ is the $\sigma$-field of subsets of $X_{\xi}$ generated by the sets in $\xi$. If $\xi=\left\{A_{0}, A_{1}, \cdots\right\}$ is a (finite or) countable partition, then any set $A \in \mathscr{F}(\xi)$ can be written as $A=\sum_{i=0}^{\infty} a_{i} A_{i}$ where $a_{i}$ is zero or one for each $i=0,1, \cdots$, with the convention that $0 \cdot B=\varnothing$ and $1 \cdot B=B$ for any set $B$.

DEFINITION 2.2. If $\xi$ is a countable partition and if $A \in \mathscr{F}$, then among the sets in $\mathscr{F}(\xi)$ there is at least one whose symmetric difference with $A$ has minimal measure. We denote by $A(\xi)$ any one of these sets.

Received by the editors March 9, 1971.

AMS 1970 subject classifications. Primary 28A65, 47A35.

Key words and phrases. Measure-preserving transformations, ergodic theory, commuting transformations.

(c) American Mathematical Society 1972 
Definition 2.3. Let $F_{n}, F \in \mathscr{F}$. We write

$$
\lim _{n \rightarrow \infty} F_{n}=F \text { if } \lim _{n \rightarrow \infty} \mu\left(F_{n} \Delta F\right)=0 .
$$

Let $\xi_{n}$ be a sequence of countable partitions. We write

for each $A \in \mathscr{F}$.

$$
\xi_{n} \rightarrow \varepsilon \text { if } \lim _{n \rightarrow \infty} A\left(\xi_{n}\right)=A
$$

Definition 2.4. In what follows, by a transformation $\tau$ (of $X$ ) we mean an invertible mapping $\tau: X \rightarrow X$ so that both $\tau$ and $\tau^{-1}$ are measure preserving. Let $\tau$ be a transformation and $\xi$ be a partition. Then $\xi$ is said to be $\tau$-admissible if $\xi=\left\{A_{0}, A_{1}, \cdots, A_{q}\right\}$ is a finite partition and if $\tau A_{i-1}=A_{i}$ for all $i=1, \cdots, q$.

Definition 2.5. A transformation $\tau$ is said to admit an approximation by partitions, if there exists a sequence $\left\{\xi_{n}\right\}$ of $\tau$-admissible partitions so that $\xi_{n} \rightarrow \varepsilon$.

Definition 2.6. If $A, B \in \mathscr{F}$ and $\varepsilon>0$, then $A=B+E(\varepsilon)$ means that $\mu(A \triangle B)<\varepsilon$.

3. Approximation of commuting transformations. Let $\tau$ and $\sigma$ be two transformations and let $s \geqq 1$ be an integer so that $\tau^{s}$ and $\sigma$ commute; i.e., $\tau^{s} \sigma x=\sigma \tau^{s} x$ for almost all $x \in X$. Also, assume that $\tau$ admits an approximation by partitions.

Lemma 3.1. Let $\xi=\left\{A_{0}, A_{1}, \cdots, A_{q}\right\}$ be a $\tau$-admissible partition. Assume that there is an integer $p \geqq 0$ so that $q=(p+1)$ s. Let $A, B \in \mathscr{F}(\xi)$, $B \cap A_{0}=\varnothing$ and $\sigma A=B+E(\varepsilon)$. Then there are $2 s$ sets $F^{r}, G^{r}, r=1, \cdots, s$, and 2 integers $m^{r}, n^{r}, r=1, \cdots, s$, so that:

(1) for each $r=1, \cdots, s, m^{r}, n^{r} \geqq 0, m^{r}+n^{r} \geqq q+1$,

(2) for each $r=1, \cdots, s, F^{r} \cap G^{r}=\varnothing$ and

$$
\begin{aligned}
& \mu\left(F^{r}\right) \leqq \frac{n^{r}+2 s}{q+1} \frac{1}{s}, \\
& \mu\left(G^{r}\right) \leqq \frac{m^{r}+2 s}{q+1} \frac{1}{s},
\end{aligned}
$$

(3) $\sigma A=\sum_{r=1}^{s}\left\{\tau^{m^{r}}\left(A \cap F^{r}\right)+\tau^{-n^{r}}\left(A \cap G^{r}\right)\right\}+E(2 \varepsilon)$.

Proof. Let $C_{i}^{r}=A_{r} \cap \sigma \tau^{i} A_{0}$. If $x \in C_{i \mid}^{r} \cap C_{j}^{r}$ then there exist two points $y$, $z \in A_{0}$ so that $x=\sigma \tau^{i} y=\sigma \tau^{j} z$ which implies that $\tau^{i-j} y=z$. Hence either $i=j$ or $|i-j| \geqq q+1$. Therefore, for a fixed $r$, any $q+1$ consecutive $C_{i}^{r}$ 's are pairwise disjoint. 
Now, let $R=X-\bigcup_{i=1}^{q} A_{i}$ and consider a fixed $l, 0 \leqq l \leqq q$. Then

$$
\begin{aligned}
\sigma A_{l} & =\sum_{r=1}^{s} \sum_{k=0}^{p} A_{k s+r} \cap \sigma A_{l}+R \cap \sigma A_{l} \\
& =\sum_{r=1}^{s} \sum_{k=0}^{p}\left[\left(\tau^{k s} A_{r}\right) \cap\left(\sigma \tau^{l} A_{0}\right)\right]+R \cap \sigma A_{l} .
\end{aligned}
$$

Since $\tau^{k s}$ commutes with $\sigma$, we then have that

or that

$$
\sigma A_{l}=\sum_{r=1}^{s} \sum_{k=0}^{p} \tau^{k s}\left[A_{r} \cap \sigma \tau^{l-k s} A_{0}\right]+R \cap \sigma A_{l}
$$

$$
\sigma A_{l}=\sum_{r=1}^{s} \sum_{k=0}^{p} \tau^{k s} C_{l-k s}^{r}+R \cap \sigma A_{l} .
$$

Hence, if $A=\sum_{l=0}^{q} a_{l} A_{l}, B=\sum_{l=0}^{q} b_{l} A_{l}$, then

$$
\begin{aligned}
\sigma A & =\sum_{l=0}^{q} a_{l} \sigma A_{l} \\
& =\sum_{l=0}^{q} \sum_{k=0}^{p} \sum_{r=1}^{s} a_{l} \tau^{k s} C_{l-k s}^{r}+R \cap \sigma A \\
& =\sum_{k=0}^{p} \sum_{r=1}^{s} b_{k s+r} A_{k s+r}+E(\varepsilon) \\
& =\sum_{k=0}^{p} \sum_{r=1}^{s} b_{k s+r} \tau^{k s} A_{r}+E(\varepsilon)
\end{aligned}
$$

or, since $B \cap R=\varnothing$,

$$
\sum_{k=0}^{p} \tau^{k s} \sum_{r=1}^{s} \sum_{l=0}^{q} a_{l} C_{l-k s}^{r}=\sum_{k=0}^{p} \tau^{k s} \sum_{r=1}^{s} b_{k s+r} A_{r}+E(\varepsilon) .
$$

Now, for a fixed $k=0, \cdots, p$ and $r=1, \cdots, s$, the sets $C_{l-k s}^{r}, l=0, \cdots, q$, are pairwise disjoint. Hence, the characteristic function of $\sum_{l=0}^{a} a_{l} C_{l-k s}^{r}$ is $\sum_{l=0}^{a} a_{l} \psi_{l-k s}^{r}$, where $\psi_{l-k s}^{r}$ denotes the characteristic function of $C_{l-k s}^{r}$. Hence

$$
\sum_{r=1}^{s} \int_{A_{r}} \sum_{k=0}^{p}\left|\sum_{l=0}^{q} a_{l} \psi_{l-k s}^{r}-b_{k s+r}\right| d \mu<\varepsilon .
$$

Therefore there exist $x_{r} \in A_{r}, r=1, \cdots, s$, so that

Hence, if

$$
\sum_{r=1}^{s} \sum_{k=0}^{p}\left|\sum_{l=0}^{q} a_{l} \psi_{l-k s}^{r}\left(x_{r}\right)-b_{k s+r}\right| \mu\left(A_{0}\right)<\varepsilon .
$$

then $\sigma A=D+E(2 \varepsilon)$.

$$
D=\sum_{r=1}^{s} \sum_{k=0}^{p} \sum_{l=0}^{q} a_{l} \psi_{l-k s}^{r}\left(x_{r}\right) A_{k s+r}
$$


Now to define the integers $m^{r}, n^{r}$ and the sets $F^{r}, G^{r}$ consider a fixed $r$, $1 \leqq r \leqq s$. Then, by a change of the summation indices one can see that

$$
\begin{aligned}
\sum_{k=0}^{p} \sum_{l=0}^{q} a_{l} \psi_{l-k s}^{r}\left(x_{r}\right) A_{k s+r}= & \sum_{u=-p s}^{(p+1) s} \psi_{u}^{r}\left(x_{r}\right) \sum_{k=0 ; 0 \leqq u+k s \leqq q}^{p} a_{u+k s} A_{r+k s} \\
= & \sum_{u=-p s}^{r-1} \psi_{u}^{r}\left(x_{r}\right) \sum_{k=0 ; 0 \leqq u+k s}^{p} a_{u+k s} A_{r+k s} \\
& +\sum_{u=r}^{(p+1) s} \psi_{u}^{r}\left(x_{r}\right) \sum_{k=0 ; u+k s \leqq q}^{p} a_{u+k s} A_{r+k s} \\
= & \sum_{u=-p s}^{r-1} \psi_{u}^{r}\left(x_{r}\right) \tau^{+(r-u)} \sum_{k=0 ; 0 \leqq u+k s}^{p} a_{u+k s} A_{u+k s} \\
& +\sum_{u=r}^{(p+1) s} \psi_{u}^{r}\left(x_{r}\right) \tau^{-(u-r)} \sum_{k=0 ; u+k s \leqq q}^{p} a_{u+k s} A_{u+k s} .
\end{aligned}
$$

Each of these two sums with respect to $u$ contains at most one nonzero term. If one of them contains all zero terms then we can define $F^{r}=\varnothing$ or $G^{r}=\varnothing$ or $F^{r}=G^{r}=\varnothing$ accordingly and see that all the conclusions of the lemma are satisfied. If both of them contain nonzero terms, corresponding, say to $u=v^{r}$ and $u=w^{r}$ with $-p s \leqq v^{r}<r, r \leqq w^{r} \leqq(p+1) s$, we then let

$$
F^{r}=\sum_{k=0 ; 0 \leqq v^{r}+k s}^{p} A_{v^{r}+k s}, \quad G^{r}=\sum_{k=0 ; w^{r}+k s \leqq q}^{p} A_{w^{r}+k s},
$$

and $m^{r}=r-v^{r}, n^{r}=w^{r}-r$. Since $w^{r}-v^{r} \geqq q+1$ it is clear that $m^{r}+n^{r} \geqq q+1$, and $m^{r}, n^{r} \geqq 0$, and that $F^{r} \cap G^{r}=\varnothing$. Also, we have that

$$
\sigma A=\sum_{r=1}^{s}\left[\tau^{m^{r}}\left(A \cap F^{r}\right)+\tau^{-n^{r}}\left(A \cap G^{r}\right)\right]+E(2 \varepsilon) .
$$

Now, to estimate the measure of $F^{r}$ and $G^{r}$, first note that the measure of each $A_{l}$ is not greater than $1 /(q+1)$. Hence, for example, $\mu\left(G^{r}\right) \leqq$ $\kappa /(q+1)$, where $\kappa$ is the number of integers in $\left\{k \mid 0 \leqq k \leqq p, w^{r}+k s \leqq q\right\}$. Then

$$
\kappa \leqq \frac{q-w^{r}}{s}+1=\frac{q-n^{r}-r}{s}+1 \leqq \frac{1-r+m^{r}}{s}+1 .
$$

Hence $\kappa \leqq m^{r} / s+2$. Therefore $\mu\left(G^{r}\right) \leqq\left(\left(m^{r}+2 s\right) /(q+1)\right)(1 / s)$. Similarly $\mu\left(F^{r}\right) \leqq\left(\left(n^{r}+2 s\right) /(q+1)\right)(1 / s)$.

THEOREM 3.1. There exist $2 s^{2}$ sequences of sets $\left\{Y_{v}^{r t}\right\},\left\{Z_{v}^{r t}\right\}$ and $2 s^{2}$ sequences of integers $\left\{m_{v}^{r t}\right\},\left\{n_{v}^{r t}\right\}$, where $\nu=1,2, \cdots$ and $r=1, \cdots, s$; $t=1, \cdots, s$, so that

$$
\sigma A=\lim _{v \rightarrow \infty} \sum_{t=1}^{s} \sum_{r=1}^{s}\left[\tau^{m_{v}{ }^{r t}}\left(A \cap Y_{v}^{r t}\right)+\tau^{-n_{v}{ }^{r t}}\left(A \cap Z_{v}^{r t}\right)\right] \quad \text { for every } A \in \mathcal{F} .
$$


Furthermore, for each $r, t=1, \cdots$, s one of the following 3 conditions holds:

(1) $\lim _{v \rightarrow \infty} \mu\left(Z_{v}^{r t}\right)=0$ and $m_{v}^{r t}=m^{r t}$ does not depend on $v$,

(2) $\lim _{v \rightarrow \infty} \mu\left(Y_{v}^{r t}\right)=0$ and $n_{v}^{r t}=n^{r t}$ does not depend on $v$,

(3) $\lim _{v \rightarrow \infty} m_{v}^{r t}=\lim _{v \rightarrow \infty} r_{v}^{r t}=\infty$.

Proof. Let $\xi_{v}=\left\{A_{0}(v), \cdots, A_{q(v)}(v)\right\}, \quad \xi_{v} \rightarrow \varepsilon$, be a sequence of $\tau$ admissible partitions. Note that $q(v) \rightarrow \infty$ as $\nu \rightarrow \infty$. Without loss of generality, we will assume that for each $\nu=1,2, \cdots$ there exists an integer $p(v)$ so that $q(v)=[p(v)+1] s$. Choose a sequence $\varepsilon_{v}>0$ so that $\varepsilon_{v} q(v) \rightarrow 0$ as $\nu \rightarrow \infty$. For each integer $\nu$ we can find another integer $\lambda=\lambda(v)$ with the following properties:

(a) there exist $B_{v}^{t}, \quad D_{v}^{t} \in \mathscr{F}\left(\xi_{\lambda(v)}\right)$ so that $A_{t}(v)=B_{v}^{t}+E\left(\varepsilon_{v}\right), \quad D_{v}^{t} \cap$ $A_{0}(\lambda(v))=\varnothing$ and $\sigma A_{t}(v)=D_{v}^{t}+E\left(\varepsilon_{v}\right)$ for every $t=1,2, \cdots, s$.

(b) $q(v) \leqq \varepsilon_{v} q(\lambda(v))$.

We then have that $\sigma B_{v}^{t}=D_{v}^{t}+E\left(2 \varepsilon_{v}\right)$. Now choose $F_{v}^{r t}, G_{v}^{r t}, m_{v}^{r t}$ and $n_{v}^{r t}$ according to the previous lemma. Hence

$$
\sigma B_{v}^{t}=\sum_{r=1}^{s}\left[\tau^{m_{v}{ }^{r t}}\left(B_{v}^{t} \cap F_{v}^{r t}\right)+\tau^{-n_{v}{ }^{r t}}\left(B_{v}^{t} \cap G_{v}^{r t}\right)\right]+E\left(4 \varepsilon_{v}\right),
$$

or

$$
\sigma A_{t}(v)=\sum_{r=1}^{s}\left[\tau^{m_{v}{ }^{r t}}\left(A_{t}(v) \cap F_{v}^{r t}\right)+\tau^{-n_{v}{ }^{r t}}\left(A_{t}(v) \cap G_{v}^{r t}\right)\right]+E\left((6+s) \varepsilon_{v}\right) .
$$

We now let

$$
Y_{v}^{r t}=\sum_{k=0}^{p(v)} \tau^{k s}\left(A_{t}(v) \cap F_{v}^{r t}\right), \quad Z_{v}^{r t}=\sum_{k=0}^{p(v)} \tau^{k s}\left(A_{t}(v) \cap G_{v}^{r t}\right) .
$$

Hence, if $A^{\prime}=\sum_{l=0}^{q(v)} a_{l} A_{l}(v) \in \mathscr{F}\left(\xi_{v}\right)$ then

$$
A=\sum_{k=0}^{p(v)} \sum_{t=1}^{s} a_{t+k s} A_{t+k s}(v)
$$

agrees with $A^{\prime}$ on all $A_{l}(v), l=1, \cdots, q(v)$, except on $A_{0}(v)$. Hence $A=A^{\prime}+E(1 / q(v))$. But

$$
\begin{aligned}
\sigma A= & \sum_{t=1}^{s} \sum_{k=0}^{p(v)} a_{t+k s} \tau^{k s} \sigma A_{t}(v) \\
= & \sum_{t=1}^{s} \sum_{k=0}^{p(v)} a_{t+k s} \tau^{k s} \sum_{r=1}^{s}\left[\tau^{m_{v}{ }^{r t}}\left(A_{t}(v) \cap F_{v}^{r t}\right)+\tau^{-n_{2}}\left(A_{t}(v) \cap G_{v}^{r t}\right)\right] \\
& \quad+E\left((6+s) q(v) \varepsilon_{v}\right) \\
= & \sum_{t=1}^{s} \sum_{r=1}^{s}\left[\tau^{m_{v}{ }^{r t}}\left(A \cap Y_{v}^{r t}\right)+\tau^{-n_{v}{ }^{r t}}\left(A \cap Z_{v}^{r t}\right)\right]+E\left((6+s) q(v) \varepsilon_{v}\right)
\end{aligned}
$$


since, for example,

Hence,

$$
\begin{aligned}
\sum_{k=0}^{p(v)} a_{t+k s} \tau^{k s} \tau^{m_{v}{ }^{r t}}\left(A_{t}(v) \cap F_{v}^{r t}\right) & =\tau^{m_{v}{ }^{r t}} \sum_{k=0}^{p(v)} a_{t+k s} \tau^{k s}\left(A_{t}(v) \cap F_{v}^{r t}\right) \\
& =\tau^{m_{v}{ }^{r t}}\left(A \cap Y_{v}^{r t}\right) .
\end{aligned}
$$

$$
\begin{aligned}
\sigma A^{\prime}= & \sum_{t=1}^{s} \sum_{r=1}^{s}\left\{\tau^{m_{v}{ }^{r t}}\left(A^{\prime} \cap Y_{v}^{r t}\right)+\tau^{-n_{v}{ }^{r t}}\left(A^{\prime} \cap Z_{v}^{r t}\right)\right\} \\
& +E\left[(6+s) q(v) \varepsilon_{v}+\left(s^{2}+1\right) / q(v)\right] .
\end{aligned}
$$

It is clear that this proves the first part of the theorem, the approximation of $\sigma A$.

To complete the proof of the theorem assume that condition (3) in the statement of this theorem is not satisfied. Then either $m_{v}^{r t}$ or $n_{v}^{r t}$ repeats an integer infinitely many times. By considering a subsequence we may assume that, for example, $m_{v}^{r t}=m^{r t}$ for all $v=1,2,3, \cdots$. But, since $F_{v}^{r t}$ and $G_{v}^{r t}$ are chosen with respect to the partition $\xi_{\lambda(v)}$, Lemma 3.1 implies that

and hence that

$$
\mu\left(G_{v}^{r t}\right) \leqq \frac{m^{r t}+2 s}{q(\lambda(v))+1} \frac{1}{s}
$$

$$
\begin{aligned}
\mu\left(Z_{v}^{r t}\right) & =\mu\left(\sum_{k=0}^{p(v)} \tau^{k s}\left(A_{t}(v) \cap G_{v}^{r t}\right)\right) \\
& \leqq(p(v)+1) \frac{m^{r t}+2 s}{q(\lambda(v))+1} \frac{1}{s} \\
& \leqq \frac{m^{r t}+2 s}{s^{2}} \frac{q(v)}{q(\lambda(v))}
\end{aligned}
$$

or, finally that $\mu\left(Z_{v}^{r t}\right) \leqq\left(\left(m^{r t}+2 s\right) / s^{2}\right) \varepsilon_{v}$. Hence $\lim _{v \rightarrow \infty} \mu\left(Z_{v}^{r t}\right)=0$.

This completes the proof of the theorem.

4. Mixing transformations with given roots. We will now apply Theorem 3.1 to show that there are strongly mixing transformations having only a finite number of specified roots. First we will note a few elementary results on transformations.

LEMMA 4.1. Let $\tau$ and $\sigma$ be two transformations of $X$. Then there exists a set $A \in \mathscr{F}$ so that $\tau(A \cap E)=\sigma(A \cap E)$ for all $E \in \mathscr{F}$ and so that $B=X-A$ is $a$ (finite or) countable union of pairwise disjoint sets $B_{i}$, satisfying $\left(\tau B_{i}\right) \cap\left(\sigma B_{i}\right)=\varnothing$ for each $i=1,2, \cdots$. Furthermore, if $\lambda$ is another transformation that commutes with both $\tau$ and $\sigma$ then $A$ is invariant under $\lambda$. 
Proof. For each $E \in \mathscr{F}$ let $A_{E}$ be the class of all measurable subsets $F$ of $E$, for which $(\tau F) \cap(\sigma F)=\varnothing$, and let $\alpha_{E}=\sup \left\{\mu(F) \mid F \in A_{E}\right\}$. If $E \in \mathscr{F}$ has a subset $G$ for which $\mu((\tau G) \triangle(\sigma G))>0$ then $F=\tau^{-1}(\tau G-\sigma G) \in A_{E}$ and $\mu(F)>0$. Therefore either $\alpha_{E}>0$ or $\tau G=\sigma G$ for all subsets $G$ of $E$.

Now construct a sequence $\left\{B_{1}, B_{2}, \cdots\right\}$ of sets as follows, using the axiom of choice.

Let $B_{1}$ be any set in $A_{X}$ for which $\mu\left(B_{1}\right) \geqq \frac{1}{2} \alpha_{X}$. If $B_{1}, B_{2}, \cdots, B_{n}$ are already chosen let $B_{n+1}$ be any set in $A_{X-\cup_{i=1}^{n} B_{i}}$ with $\mu\left(B_{n+1}\right) \geqq$ $\frac{1}{2} \alpha_{X-\cup_{i=1}^{n} B_{i}}$. Then $B_{n}$ 's are pairwise disjoint and $\left(\tau B_{n}\right) \cap\left(\sigma B_{n}\right)=\varnothing$ for each $n=1,2, \cdots$. Let $B=\bigcup_{n=1}^{\infty} B_{n}$ and $A=X-B$. We will now show that $\alpha_{A}=0$. In fact, since $A \subset X-\bigcup_{i=1}^{n} B_{i}$ for all $n \geqq 1, \alpha_{A} \leqq \alpha_{X-\cup_{i=1}^{n} B_{i}} \leqq 2 \mu\left(B_{n+1}\right)$ and $\mu\left(B_{n}\right) \rightarrow 0$, for $B_{n}$ 's are pairwise disjoint and $\mu(X)<\infty$.

Now let $\lambda$ be a transformation which commutes with $\tau$ and $\sigma$. Let $A^{\prime}=\lambda A$. Then $\tau\left(A^{\prime} \cap E\right)=\tau \lambda\left(A \cap \lambda^{-1} E\right)=\lambda \tau\left(A \cap \lambda^{-1} E\right)=\lambda \sigma\left(A \cap \lambda^{-1} E\right)=$ $\sigma\left(A^{\prime} \cap E\right)$ for any $E \in \mathscr{F}$. Hence $\alpha_{A^{\prime}}=0$. But this implies that $\mu\left(B \cap A^{\prime}\right)=0$, since any nonnull subset of $B$ has a positive $\alpha$. Therefore $A$ is invariant under $\lambda$.

LEMMA 4.2. Let $\tau$ and $\sigma$ be two transformations that satisfy the conditions in §3. Assume that $\tau^{s}$ is ergodic. Then there exists an approximation of $\sigma$ as given in Theorem 3.1, for which either $\lim _{v \rightarrow \infty} m_{v}^{r t}=\lim _{v \rightarrow \infty} n_{v}^{r t}=\infty$ for each $r, t=1, \cdots, s$ or $\sigma=\tau^{p}$ for some integer $p$.

Proof. Consider the approximation of $\sigma$ as given in Theorem 3.1 and assume that $\lim _{v \rightarrow \infty} m_{v}^{r t}=\lim _{v \rightarrow \infty} n_{v}^{r t}=\infty$ is not satisfied for some $r_{0}, t_{0}$. Then either (1) or (2) of Theorem 3.1 holds. We will only consider the case where (1) holds; the other case is similar. Hence $m_{v}^{r_{0} t_{0}}=p$ for some integer $p$ and $\lim _{v \rightarrow \infty} \mu\left(Z_{v}^{r_{0} t_{0}}\right)=0$. If $\lim _{v \rightarrow \infty} \mu\left(Y_{v}^{r_{0} t_{0}}\right)=0$, then the term corresponding to $r_{0} t_{0}$ does not play any role in the approximation of $\sigma$ and the corresponding powers $m_{v}^{r_{0} t_{0}}, n_{v}^{r_{0} t_{0}}$ can be changed arbitrarily without disturbing the form of approximation. Hence, in this case we can find a new approximation for $\sigma$ for which $\lim _{v \rightarrow \infty} m_{v}^{r_{0} t_{0}}=\lim _{v \rightarrow \infty} n_{v}^{r_{0} t_{0}}=\infty$. Now assume that $\lim \sup _{v \rightarrow \infty} \mu\left(Y_{v}^{r_{v} t_{0}}\right)>0$. In this case we will show that $\sigma=\tau^{p}$.

In fact, let $A$ and $B=\bigcup_{n=1}^{\infty} B_{n}$ be the decomposition of $X$ as given in Lemma 3.1, corresponding to the automorphisms $\tau^{p}$ and $\sigma$. Since $\tau^{s}$ commutes both with $\tau^{p}$ and $\sigma$, Lemma 4.1 gives that $A$ is invariant under $\tau^{s}$. Hence either $A=X$ or $B=X$, for $\tau^{s}$ is ergodic. If $A=X$, then $\sigma E=\tau^{p} E$ for all $E \in \mathscr{F}$ and hence $\sigma=\tau^{p}$ as is well known.

If $B=X$, since lim sup $\operatorname{sum}_{v \rightarrow \infty} \mu\left(Y_{v}^{r_{v} t_{0}}\right)>0$, then there exists a $C=B_{i}$, so that $\lim \sup _{v \rightarrow \infty} \mu\left(Y_{v}^{r_{0} t_{0}} \cap C\right)>0$ and $(\sigma C) \cap\left(\tau^{p} C\right)=\varnothing$. But then it is clear that

$$
\sum_{r=1}^{s} \sum_{t=1}^{s}\left[\tau^{m_{v}{ }^{r t}}\left(C \cap Y_{v}^{r t}\right)+\tau^{-n_{v}{ }^{r t}}\left(C \cap Z_{v}^{r t}\right)\right]
$$


fails to converge to $\sigma C$. This is a contradiction and completes the proof.

To proceed, we will now quote a result of Ornstein [5], without proof.

THEOREM 4.1. There exists a strongly mixing automorphism that admits an approximation by partitions.

Recall that an automorphism $\tau$ of $X$ is called strongly mixing if $\lim _{v \rightarrow \infty}\left(\tau^{p} E \cap F\right)=\mu(E) \mu(F)$ for each $E, F \in \mathscr{F}$.

THEOREM 4.2. Let $\tau$ be a strongly mixing automorphism that admits an approximation by partition and let $s \geqq 1$ be an integer. If an automorphism $\sigma$ commutes with $\tau^{s}$ then $\sigma$ is a power of $\tau$; i.e., there exists an integer $p$ so that $\sigma=\tau^{p}$.

Proof. We apply Lemma 4.2. If $\sigma$ is not a power of $\tau$, then

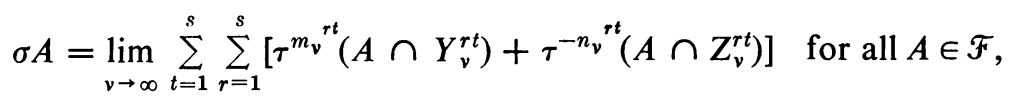

where $\lim _{v \rightarrow \infty} m_{v}^{r t}=\lim _{v \rightarrow \infty} n_{v}^{r t}=\infty$ for each $r, t=1, \cdots, s$. Hence

$$
\sigma A \subset \sum_{t=1}^{s} \sum_{r=1}^{s}\left[\tau^{m_{v}{ }^{r t}} A+\tau^{-n_{v}{ }^{r t}} A\right]+E_{v} \quad \text { where } \quad \mu\left(E_{v}\right) \rightarrow 0 \text { as } \nu \rightarrow \infty
$$

Therefore

$$
\begin{aligned}
\sigma A \subset \sum_{r, t, k, j=1}^{s} & \left\{\left(\tau^{m_{l}{ }^{k j}} A\right) \cap\left(\tau^{m_{v}{ }^{r t}} A\right)+\left(\tau^{m_{l}{ }^{k j}} A\right) \cap\left(\tau^{-n_{v}{ }^{r t}} A\right)\right. \\
& \left.+\left(\tau^{-{ }{ }^{k j}} A\right) \cap\left(\tau^{m_{v}}{ }^{r t} A\right)+\left(\tau^{-n_{l}}{ }^{k j} A\right) \cap\left(\tau^{-n_{v}{ }^{r t}} A\right)\right\}+E_{v l}
\end{aligned}
$$

where $\mu\left(E_{v l}\right) \rightarrow 0$ as $\nu, l \rightarrow \infty$. From this and the strong mixing of $\tau$ it follows that $\mu(A) \leqq s^{4}[\mu(A)]^{2}$ for all $A \in \mathscr{F}$, which is impossible.

This theorem implies that $\tau^{s}$ has an $n$th root if and only if $n$ divides $s$. Therefore we have the following result.

COROLLARY. There exists a strongly mixing automorphism that has roots in a finitely generated class of integers and not in the complement of the class.

\section{BIBLIOGRAPHY}

1. M. A. Akcoglu, R. V. Chacon and T. Schwartzbauer, Commuting transformations and mixing, Proc. Amer. Math. Soc. 24 (1970), 637-642. MR 40 \#7421.

2. R. V. Chacon and T. Schwartzbauer, Commuting point transformations, Z. Wahrscheinlichkeitstheorie und Verw. Gebiete 11 (1969), 277-287. MR 39 \#2939.

3. R. V. Chacon, A geometric construction of measure preserving transformations, Proc. Fifth Berkeley Sympos. Math. Statist. and Probability (Berkeley, Calif., 1965/66), vol. II: Contributions to Probability Theory, part 2, Univ. of California Press, Berkeley, Calif., 1967, pp. 335-360. MR 35 \#3033. 
4. N. Friedman, Introduction to ergodic theory, Van Nostrand Math. Studies, New York, 1970.

5. D. S. Ornstein, A mixing transformation that commutes only with its powers, Proc. Sixth Berkeley Sympos. Math. Statist. and Probability, vol. II, part 2, Univ. of California Press, Berkeley, Calif., 1970, pp. 335-360.

University of Toronto, Toronto 181, Ontario, Canada

University of Minnesota, Minneapolis, Minnesota 55455 